\title{
Preliminary results of a randomized crossover clinical trial comparing a new electromagnetic and vibrating device and alpha-blocker agents in patients affected by bladder outlet obstruction secondary to benign prostatic hyperplasia
}

\author{
Simone Brardi ${ }^{1}$, Giuseppe Romano ${ }^{2}$, Gabriele Cevenini ${ }^{3}$ \\ ${ }^{1}$ Hemodialysis Unit, S. Donato Hospital, Arezzo, Italy; \\ ${ }^{2}$ Urology Unit, S. Maria della Gruccia Hospital, Montevarchi, Italy; \\ ${ }^{3}$ Department of Medical Biotechnologies, University of Siena, Italy.
}

KEY WORDS: Benign prostatic hyperplasia; Pulsed electromagnetic fields, Alpha-bocker agents; Randomized crossover clinical trial.

Submitted 27 August 2021; Accepted 19 September 2021

To the Editor,

Benign Prostatic Hyperplasia (BPH) is one of the main causes of patients seeking urological counselling in Western countries. It has been estimated that nearly 70 percent of United States men between the ages of 60 and 69 years, and nearly 80 percent of men $\geq 70$ years, have some degree of $\mathrm{BPH}(1)$.

BPH is a histologic diagnosis defined as an increase in the total number of stromal and glandular epithelial cells within the transition zone of the prostate gland. BPH results in Benign Prostatic Enlargement (BPE) that can, in turn, lead to Bladder Outlet Obstruction (BOO). BPE and mostly BOO is often associated with Lower Urinary Tract Symptoms (LUTS), which can be subdivided into symptoms of urinary storage (eg, urgency, daytime frequency, nocturia, incontinence, etc.), symptoms of urinary voiding (eg, slow stream, intermittent stream or intermittency, hesitancy, straining to void, terminal dribble, dysuria, etc.) and post-voiding symptoms (eg, sensation of incomplete bladder emptying, post-void urinary dribbling, etc.). All above-mentioned symptoms reported by patients with BPH contribute to a significantly reduced quality of life $(2,3)$.

The initial treatment for Bladder Outlet Obstruction (BOO) secondary to Benign Prostatic Hyperplasia (BPH) is generally pharmacologic, especially in patients with mild to moderate symptoms and no clear indication for surgical intervention. Medical therapy consists of alpha-blockers, 5-alpha reductase inhibitors, or a combination of these agents (4). Alphablockers are first-line agents used for the treatment of symptomatic BPH. These drugs relax the smooth muscle tone at the bladder neck. If associated with 5-alpha reductase inhibitor drugs the use of alpha-blockers agents may result more effective than monotherapy with either drug alone (5).

Given the above, several data in the literature reported that electromagnetic fields (EMF) have many biological activities capable of interfering with the ability to reproduce and differentiate cells, modulating the inflammatory system through the increase of oxide-reductive potential, and increasing microvascular motility, ATP production, hormonal secretion, antioxidant enzyme activity, and cellular metabolism (6); moreover EMF at high frequency and low intensity allows to obtain significant therapeutic results without unwanted side effects, allowing their use also in a wide spectrum of chronic diseases characterized by functional disorders and pain, such as chronic inflammatory diseases (7).

The antiphlogistic and stimulating effects of the tissue repair produced by magnetic fields in humans allows to achieve favorable therapeutic results especially in diseases affecting the osteoskeletal system, such as the fractures and the arthropathies (8).

The vibrating systems are equipment capable of generating sinusoidal oscillations at various frequencies and transfer them to the body of the subject to be treated through pressure waves, with specially designed platforms just capable of vibrating at variable frequencies (Hertz/sec) (9).

The treatment with vibrations exerts a safe myo-relaxant effect with consequent reduction of muscle spasticity and is widely used in the field of neuro-rehabilitation (10). With vibratory frequencies varying between 5 and $30 \mathrm{~Hz}$, an increase in cerebral cortisone and serotonin has been demonstrated in the rat; in humans, mono or polysynaptic connections are activated in order to generate reflex contractions (11).

The STIM-PLAVIM ${ }^{\circledR}$, a device which is capable of simultaneously generating an intense variable electromagnetic field and vibratory stimulation, was object of an observational perspective study in patients affected by voiding symptoms attributed to bladder outlet obstruction secondary to benign prostatic hyperplasia and already treated by alpha-blocker agents. These

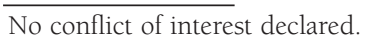


patients without stopping the pharmacologic therapy added the application of the STIM-PLAVIM ${ }^{\circledR}$ device at the perineal level every day for 30 minutes for a period of 60 days.

The application of the above mentioned device resulted in a rapid improvement of voiding symptoms as confirmed by the statistically significant reduction in the mean score of the International Prostatic Symptoms Score (IPPS) (12). However, the above mentioned study was performed in an uncontrolled design hence the need to compare the STIM-PLAVIM ${ }^{\circledR}$ with the use of alpha-blocker agents which today represent the cornerstone of the current standard therapy for bladder outlet obstruction secondary to BPH (4). We hypothesized that, in the above-mentioned patients the use of STIM-PLAVIM ${ }^{\circledR}$ device would be more effective than alpha-blockers in reducing the mean volume of bladder residue after voiding. We further hypothesized that the use of STIM-PLAVIM ${ }^{\circledR}$ device would improve secondary parameters tied to BPH such as the prostatic volume, the total PSA value and the IPSS more than conventional alpha-blockers agents therapy.

\section{MATERIAL AND METHODS}

This study was performed in a monocentric, controlled, randomized, two-periods crossover design.

Each period included 45 daily applications of STIM-PLAVIM ${ }^{\circledR}$ device at the perineal level each lasting 30 minutes without the intake of any alpha- blocker agent (although 5-alpha reductase inhibitors, mainly dutasteride and secondarily finasteride, were used in $60 \%$ of cases) or the daily oral intake of an alpha-blocker agent (60\% alfuzosin and tamsulosin for the rest) for a same length of 45 days. When already in use the 5-alpha reductase inhibitors were associated with the alpha-blocker agents too and therefore the use of 5-alpha reductase inhibitors remained unchanged in both periods. At crossover and at the end of the study assessment were done. Patients were randomly assigned to start the study with a period of daily STIM-PLAVIM ${ }^{\circledR}$ device application or a period of daily oral intake of an alpha-blocker agent using a computer-generated list. Masking the treatment allocation for physician and patients was not feasible. Ten ambulatory patients (mean age $65 \pm 7$ years) affected by bladder outlet obstruction secondary to BPH were enrolled. All patients provided written informed consent before participating. The inclusion criteria were a clinical, laboratory and ultrasound diagnosis of benign prostatic hyperplasia at least 6 months prior and age between 50 and 80 years. Exclusion criteria were a proven diagnosis of prostatic cancer, urinary infections, the permanent or temporary use of bladder catheter, neurological voiding disturbances, alpha-blockers agents intolerance or the presence of medical electrical devices incompatible with the use of electromagnetic fields. The volume of bladder residue after voiding was estimated with a suprapubic ultrasound method as well as the prostatic volume was assessed by a transrectal ultrasound method in both cases using the same ultrasound device Logiq S7 (GE Medical Systems Italy S.P.A. Milan, Italy) sonographic system equipped with 3 to $5 \mathrm{Mhz}$ convex transducers for the suprapubic ultrasound and a BE9CS (GE Medical Systems Italy S.P.A. Milan, Italy) Biplane intracavity probe with a bandwidth of 4-11 Mhz for the transrectal ultrasound. All ultrasonography examinations were carried out by the same nephrologist experienced in ultrasound examination. To characterize the subjective evaluation of the urological symptoms, the International Prostatic Symptoms Score (IPSS) was assessed and finally was tested the total PSA value. The STIM-PLAVIM ${ }^{\circledR}$ device used in our study is a Medical Device consisting of a polyethylene/erthalite container hand piece, washable and sterilizable, consisting of two cylinders: the larger one contains a low voltage electric motor inside, which is connected by a joint to a shaft in aluminum/stainless steel with a modulating speed motor. The shaft containing the motor has the shape of a cradle able to house the high-quality neodymium magnet which exerts an intense magnetic force between 1000 and 1500 Gauss. The housing of the magnet in the cradle is assembled asymmetrically so that the rotation of the motor, with adjustable speed by means of a special dedicated electronic program, allows to simultaneously generate an intense permanent and rotating magnetic field and a vibratory trend with specific frequency (between 80 and $140 \mathrm{Hertz}$ ). The handpiece, with an ergonomic cylindrical shape, is closed at the two lower and upper ends with two caps with screw closure; the upper part of the Device also contains a rechargeable electric accumulator (with a common 220 Volt electric socket) (Figures 1, 2).

\section{Statistical analysis}

Quantitative variables, pre- and post-treatment with STIM-PLAVIM ${ }^{\circledR}$ device, were tested for their mean reduction using Student's t-test for paired data, before testing for normality with the Kolmogorov-Smirnov test. A significance level of 95\% ( $\mathrm{p}<0.05$ ) was chosen for all statistical analyses performed with SPSS software, version 10.

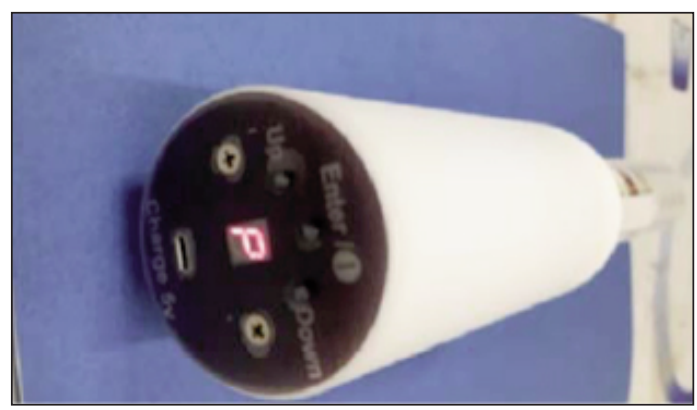

Figure 1.

STIM-PLAVIM ${ }^{\circledR}$ device.
Figure 2. STIM-PLAVIM ${ }^{\circledR}$ device application modality.

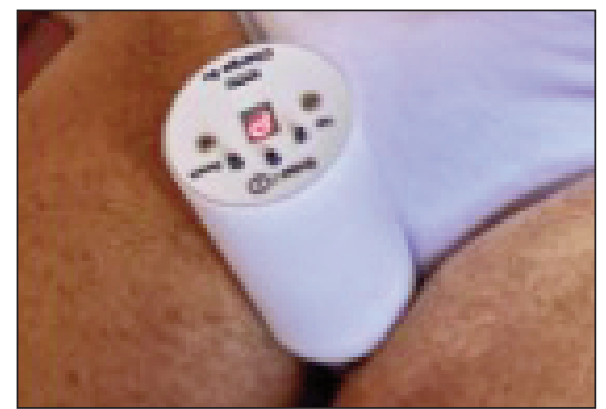




\section{RESULTS}

Statistically significant reductions in prostate volume, postvoid residual bladder volume and suprapubic prostatic transverse diameter between pre- and post-treatment with STIM-PLAVIM ${ }^{\circledR}$ device were found while there was no statistically significant reduction in PSA and IPSS. Prostatic volume, postvoid residual bladder volume, suprapubic prostatic transverse diameter, total PSA and IPSS before and after application of STIM-PLAVIM ${ }^{\circledR}$ device are shown in Table 1 . The statistical results of the comparison between pre- and post-treatment data with STIM-PLAVIM ${ }^{\circledR}$ device are instead shown in Table 2.

Reductions in prostate volume (mean difference of $6.35 \mathrm{ml}$, $\mathrm{p}=0.003$ ), postvoid residual bladder volume (mean difference of $28.8 \mathrm{ml}, \mathrm{p}=0.032$ ) and suprapubic prostatic transverse diameter (mean difference of $4 \mathrm{~mm}, \mathrm{p}=0.001$ ) were significant at $95 \%$, while there was no statistically significant reduction in PSA and IPSS. No problems related to adverse reactions or intolerance neither with the oral intake of alpha-blocker agents neither with application of STIMPLAVIM $^{\circledR}$ device were reported. All patients completed the planned two-periods of the study, and none was excluded because of intolerance or lack of compliance.

\section{Discussion}

The main finding of this study is that STIM-PLAVIM ${ }^{\circledR}$ device significantly reduced, in BPH patients, prostate volume and postvoid bladder volume more than the intake of any alpha-blocker agent as well as the suprapubic prostatic transverse diameter. Instead, there was no statistically significant reduction in total PSA and IPSS.

These results are partially consistent with the results of previous studies such as that one of Elgohary et al. (13) which demonstrated the effectiveness of pulsed electromagnetic fields alone or in association to exercise therapy in the treatment of benign prostatic hypertrophy in terms of IPSS improvement and reduction of bladder residue after voiding and that of Tenuta et al. (14) which likewise demonstrated the effectiveness of pulsed electromagnetic fields in terms of prostate volume reduction and IPSS enhancement.

In neither of the two studies however the pulsed electromagnetic fields were compared with the standard drug therapy for benign prostatic hypertrophy.

In the study of Giannakopoulos et al. (15) the exposure of BPH patients to a pulsed electromagnetic field or an alpha blocker therapy resulted in a statistically significant decrease of prostate volume and bladder residue after voiding, in the pulsed electromagnetic fields group, while the IPSS showed a statistically significant decrease before and after treatment both with pulsed electromagnetic fields and with the use of alpha-blockers.

All above studies however used pulsed electromagnetic fields devices without the vibratory stimulus that is present in the STIM-PLAVIM ${ }^{\circledR}$ device.

About lack of improvement in IPSS with STIM-PLAVIM ${ }^{\circledR}$ device it is necessary to point out that are well known the changes in the IPSS obtained with dutasteride, tamsulosin, and combination therapy in men with symptomatic benign prostatic hyperplasia and an enlarged prostate because the combination therapy with tamsulosin and dutasteride affords the greatest and the most rapid symptomatic benefit among those men and is effective regardless of previous BPH medical therapy (16). Given the above, the performance of STIM-PLAVIM ${ }^{\circledR}$ device about IPSS, similarly to what found in the work of Giannakopoulos (15), represents an invariance result rather than a failure since this result was obtained in comparison with alpha blockers that are very effective in ameliorating the IPSS.

Besides, the failure to reduce IPSS remained within clinically insignificant limits and may be certainly compensated by the advantages in terms of greater safety, since all the adverse effects usually associated with alpha-blockers were excluded and there was no notable adverse effect with the use of STIM-PLAVIM ${ }^{\circledR}$ device nor significant contraindications to its use which resulted always well tolerated. Furthermore STIM-PLAVIM ${ }^{\circledR}$ device proved to be safe and more effective than alpha-blockers with respect to quantitative and objective parameters such as prostate volume and bladder residue after voiding. About the invariance of total PSA we must point out that in the our study the daily oral intake of an alpha-blocker agent or the use of STIM-PLAVIM ${ }^{\circledR}$ device were associated with a wide use of 5-alpha reductase inhibitors whose use remained therefore unchanged throughout the all duration of the study, so that we believe that the use of 5-alpha reductase inhibitors may be cause of the invariance of the total PSA values since those drugs have a deep impact over the total PSA values (16). 


\section{Conclusions}

The our study, despite the small scale of the sample examined, it is the first pulsed electromagnetic fields study provided in a controlled, randomized and crossover design in patients affected by bladder outlet obstruction secondary to BPH where the pulsed electromagnetic fields (with the vibratory stimulus that is present in the STIM-PLAVIM ${ }^{\circledR}$ device) were compared with the standard BPH drug therapy obtaining a statistically significant decrease of two objective and quantitative parameters as the prostate volume and the postvoid residual bladder volume. This study therefore may open to a clinical use on a larger scale of this device in the same patients affected by bladder outlet obstruction secondary to BPH.

\section{REFERENCES}

1. McVary KT. Epidemiology and pathophysiology of benign prostatic hyperplasia. In: UpToDate, post TW (Ed), UpToDate, Waltham, MA. 2020.

2. McVary KT. Lower urinary tract symptoms in men. In: UpToDate, post TW (Ed), UpToDate, Waltham, MA. 2020.

3. Fourcade RO, Lacoin F, Rouprêt M, et al. Outcomes and general health-related quality of life among patients medically treated in general daily practice for lower urinary tract symptoms due to benign prostatic hyperplasia. World J Urol. 2012; 30:419-426.

4. Gratzke C, Bachmann A, Descazeaud A, et al. EAU Guidelines on the assessment of non-neurogenic male lower urinary tract symptoms including benign prostatic obstruction. Eur Urol. 2015; 67:1099-1109.

5. Roehrborn CG, Siami P, Barkin J, et al. For the CombAT Study Group. The effects of dutasteride, tamsulosin and combination therapy on lower urinary tract symptoms in men with benign prostatic hyperplasia and prostatic enlargement: 2-year results from the CombAT study. J Urol. 2008; 179:616-621.

6. BinhiV, Savin A. Effects of weak magnetic fields on biological systems: physical aspects, Phys Us pekhi. 2003; 46:259-291.

7. McFarlane JP, Foley SJ, de Winter P, et al. Acute suppression of idiopathic detrusor instability with magnetic stimulation of the sacral nerve roots. Br J Urol. 1997; 80:734-741.

8. Haddad JB, Obolensky AG, Shinnick P. The biologic effects and the therapeutic mechanism of action of electric and electromagnetic field stimulation on bone and cartilage: New findings and a review of earlier work. J Altern Complement Med. 2007; 13:485-490.

9. Bosco C, Cardinale M. Nuove frontiere dell'allenamento sportivo: le vibrazioni. Effetti sul comportamento meccanico del muscolo scheletrico. Coaching and Sport Science Journal. 1998; 3:53-59.

10. Flieger J, Karachalios T, Khaldi L, et al. Mechanical stimulation in the form of vibration prevents postmenopausal bone loss in ovariectomized rats. Calcif Tissue Int. 1998; 63:510-514.

11. Rodrigues MP, Paiva LL, Ramos JGL, Ferla L. Vibratory perineal stimulationfor the treatment of female stress urinary incontinence: A systematic review. Int Urogynecol J. 2018; 4:555-562.

12. Brardi S, Biandolino P, Giovannelli V, et al. Possible applications of electromagnetic fields in the treatment of symptoms related to benign prostatic hyperplasia. Am J Urol Res. 2020; 5:006-010.

13. ElgoharyHM, Tantawy SA. Pulsed electromagnetic field with or without exercise theraphy in the treatment of benign prostatic hyperplasia. J Phys Ther Sci. 2017; 29:1305-1310.

14. Tenuta M, Tarsitano MG, Mazzotta $P$, et al. Therapeutic use of pulsed electromagnetic field therapy reduces prostate volume and lower urinary tract symptoms in benign prostatic hyperplasia. Andrology. 2020; 8:1076-1085.

15. Giannakopoulos XK, Giotis C, karkabounas S Ch, et al. Effects of pulsed electromagnetic fields on benign prostate hyperplasia. Int Urol Nephrol. 2011; 43:955-60.

16. Roehrborn CG, Siami P, Barkin J, et al. The influence of baseline parameters on changes in international prostate symptom score with dutasteride, tamsulosin, and combination therapy among men with symptomatic benign prostatic hyperplasia and an enlarged prostate: 2-year data from the combat study. Eur Urol. 2009; 55:461-471.

\section{Correspondence}

Simone Brardi, MD (Corresponding Author)

sibrardi@gmail.com

Hemodialysis Unit, S. Donato Hospital, Arezzo (Italy)

Giuseppe Romano, MD

giuseppe.romano@uslsudest.toscana.it

Urology Unit, S. Maria della Gruccia Hospital, Montevarchi (Italy)

Gabriele Cevenini, MD

gabriele.cevenini@unisi.it

Department of Medical Biotechnologies, University of Siena (Italy) 